\title{
Amenorrhea after chemoembolization and suction curettage of caesarean scar pregnancy
}

\author{
Piotr Czuczwar, Anna Stępniak, Piotr Szkodziak, Wojciech Wrona, Sławomir Woźniak \\ $3^{\text {rd }}$ Department of Gynecology, Lublin, Poland
}

\section{LEARNING OBJECTIVES}

To better understand the management of caesarean scar pregnancy (CSP) and to be able to recognize and to manage a potential complication of an intervention used to treat CSP.

CSP is a rare location of ectopic pregnancy implanted in the area of the previous caesarean section (CS) scar. CSP is associated with a high risk of haemorrhage and in severe cases even a necessity to perform life saving hysterectomy. Selective uterine artery chemoembolization with intra-arterial methotrexate (MTX) infusion followed by suction curettage is one of the treatment options available for CSP. Literature data on the possible complications of this treatment approach are scarce.

\section{CASE PRESENTATION}

A 35-year-old female of Caucasian racial origin, one CS in her obstetrical history, was admitted to the hospital due to suspicion of CSP. Transvaginal ultrasound examination confirmed the initial diagnosis. The patient was qualified for chemoembolization of uterine arteries and suction curettage of post cesarean scar implantation site. No complications were observed during these procedures. Four months after initial treatment the patient reported amenorrhea and pelvic pain. The suspicion of Asherman's syndrome (AS) was considered and confirmed by transvaginal ultrasound (Fig. 1) and sonohysterography. The patient wished to preserve fertility and because of this was qualified for hysteroscopy and simultaneous laparoscopy. Intrauterine adhesions were visualized (Fig. 2) and deliberated (Fig. 3). Finally, chromopertubation confirmed the patency of both fallopian tubes. To prevent the recurrence of adhesions a copper containing intrauterine device (IUD) was inserted. Normal menses occurred in this woman 35 days after the second stage of treatment. Intrauterine cavity location of the IUD was confirmed by transvaginal ultrasound (Fig. 4).

The diagnosis of AS requires documentation of intrauterine adhesions in addition to clinical features such as amenorrhea, hypomenorrhea, subfertility or recurrent pregnancy loss. Transvaginal ultrasound is the first-line imaging method. The diagnosis may be confirmed by sonohysterography and, ultimately, during hysteroscopy. The true prevalence of the reported complication is unknown, but ranges from $2 \%$ to $48 \%$ in prospective series of women following surgical evacuation of an early pregnancy loss. While dilatation and

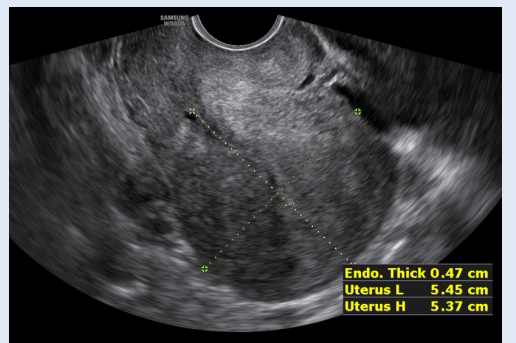

Figure 1. Four months after chemoembolization and suction curettage of CSP transvaginal ultrasound examination performed in the luteal phase of the menstrual cycle revealed narrow endometrium with hypoechoic bands, suggestive of intrauterine adhesions in AS

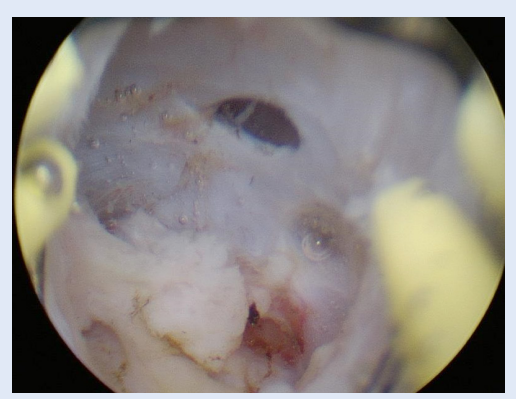

Figure 2. During hysteroscopy adhesions were seen within the entire uterine cavity, the diagnosis of AS was confirmed

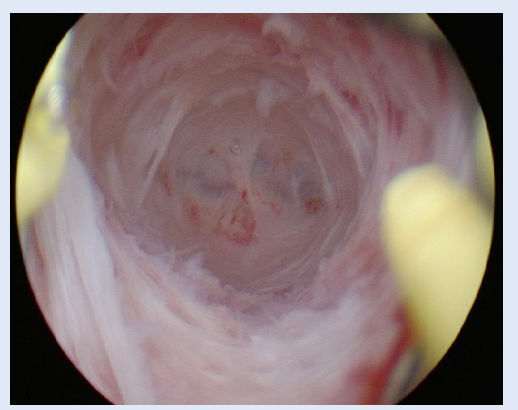

Figure 3. Hysteroscopic image of the uterine cavity after adhesiolysis 
curettage with a sharp curette is a known risk factor for AS, there are no reports of AS following suction curettage. In our case the chemoembolization could have contributed to the formation of intrauterine adhesions. Currently, the recommended treatment of AS is hysteroscopic deliberation of adhesions.

To the best of our knowledge there are no reported cases of AS after chemoembolization and suction curettage of CSP. Given the rarity of the presented case, the patients qualified for this procedure should be informed about the possible risk of the above complication. We believe that the physicians should be aware of the possibility of AS following treatment of CSP, especially if amenorrhea develops after the intervention.

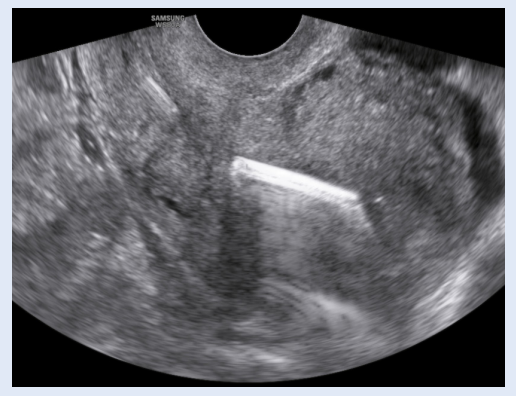

Figure 4. Five weeks after hysteroscopic adhesiolysis the proper position of the IUD inserted to prevent recurrence of adhesions was confirmed by transvaginal ultrasound 